\title{
Microscopic structure factor of liquid parahydrogen: Monte Carlo and molecular dynamics simulations
}

\author{
F. J. Bermejo, ${ }^{1,2}$ B. Fåk, ${ }^{3}$ S. M. Bennington, ${ }^{3}$ K. Kinugawa, ${ }^{4}$ J. Dawidowski, ${ }^{5}$ M. T. Fernández-Díaz, ${ }^{6}$ C. Cabrillo, ${ }^{1}$ \\ and R. Fernández-Perea ${ }^{1}$ \\ ${ }^{1}$ Consejo Superior de Investigaciones Científicas, Serrano 123, E-28006 Madrid, Spain \\ ${ }^{2}$ Department of Electricity and Electronics, University of the Basque Country, P.O. Box 644, Bilbao 48080, Spain \\ ${ }^{3}$ ISIS Facility, Rutherford Appleton Laboratory, Chilton, Didcot, Oxon OX11 0QX, United Kingdom \\ ${ }^{4}$ Department of Chemistry, Nara Womens University, Nara 630-8506, Japan \\ ${ }^{5}$ Centro Atómico Bariloche, 8400 San Carlos de Bariloche, Rio Negro, Argentina \\ ${ }^{6}$ Institut Laue Langevin, Boîte Postale 156x, F-38042 Grenoble Cedex 9, France
}

(Received 1 March 2002; revised manuscript received 17 June 2002; published 31 December 2002)

\begin{abstract}
We present a comparison of results on the microscopic structure of liquid parahydrogen as calculated by path-integral Monte Carlo and path-integral-centroid-molecular-dynamics simulations. The radial distribution functions calculated using both approaches are found to be in good agreement. The disagreement between published estimates for the static structure factor are found to arise from different approximations followed for the Fourier transform of heavily truncated data. A comparison of the structure of the real liquid with that of a classical analog is also made and shows that the latter would freeze at the experimental liquid density. Liquid parahydrogen is therefore stabilized by the action of large quantum effects.
\end{abstract}

DOI: 10.1103/PhysRevB.66.212202

PACS number(s): 67.20.+k, 61.12.-q, 61.20.Ja

The continued interest on the properties of liquid hydrogen stems from different reasons. First, it is known to be one of the dominant constituents of the giant planets ${ }^{1}$ where it is found in various states of aggregation stretching over a vast range of densities and temperatures. Second, laboratory efforts to cross the insulator $\rightarrow$ metal transition resulting in the production of metallic hydrogen continue apace ${ }^{2}$ and finally, apart from its use as a cryogenic liquid or as a fuel element in spacecraft technology, the material still constitutes a promising energy source since it is environmentally safe and has a high caloric content.

The fundamental difficulties in dealing with this liquid arise from the light masses of its constituent particles and the relatively low temperatures where the liquid exists under its saturated vapor pressure. This makes quantum effects prominent and its first manifestation is the appearance of a discrete spectrum of transitions between molecular rotational levels. The quantum nature of such motions imposes some symmetry constraints to the total molecular wave function. This means that the rotational states and the nuclear spin states of the two protons forming the $\mathrm{H}_{2}$ molecule are not independent. Coupling of nuclear spin states $(I=0$ for a molecule having antiparallel proton spins and $I=1$ for parallel spin states) leads to two distinguishable species, para $-\mathrm{H}_{2}\left(p-\mathrm{H}_{2}\right)$ and ortho $-\mathrm{H}_{2}\left(o-\mathrm{H}_{2}\right)$, respectively. This results in special characteristics of the interaction potential between $\mathrm{H}_{2}$ molecules. Such constraints imply that $p-\mathrm{H}_{2}$ molecules interact with its neighbors through an isotropic potential since the total wave function, and therefore the electronic charge distribution, will have spherical symmetry, whereas $o-\mathrm{H}_{2}$ shows a strong angular dependence of such interactions due to the action of a finite electric quadrupole moment.

The liquid structure function of $p-\mathrm{H}_{2}$ as quantified by the $g(r)$ radial distribution function is now beginning to be understood mostly by recourse to computer simulations where the quantum degrees of freedom are explicitly taken into consideration. A preliminary report on results from pathintegral-centroid-molecular-dynamics (PICMD) Simulations (Ref. 3) has shown that the liquid is significantly structured, showing well defined oscillations appearing in its $g(r)$ up to relatively long distances. On the other hand, derivation of structural information by experimental means such as neutron diffraction is hampered by a number of reasons that arise from the strong energy dependence of the neutron cross section of $p-\mathrm{H}_{2},{ }^{4}$ as it is known since the early days of neutron scattering. First and foremost, the usual approximation, upon which diffraction studies on liquids rely upon, that relates the observed total cross section

$$
\Sigma_{Q} \propto S_{m}(Q)=f_{1}(Q)+D_{m}(Q)
$$

to the sought liquid structure factor $D_{m}(Q)$ plus an individual molecule form factor $f_{1}(Q)$ is here of little value. To see this let us consider the response to neutron irradiation of a sample in thermal equilibrium close to the triple point. At $T=15 \mathrm{~K}$ its equilibrium composition ${ }^{5} p_{K}$ yields 0.9991 for $p-\mathrm{H}_{2}$, the rest being orthohydrogen. Rotational para $\rightarrow$ ortho transitions are separated by energies $E_{K K^{\prime}}=B_{e} K(K+1)$ with a rotational constant $B_{e}=7.35 \mathrm{meV}$. Transitions connecting states with increasing values of the rotational quantum number $K$ can be induced by the magnetic moment of the incoming neutron. This means that such sample will change its $p / o$ ratio if irradiated by a neutron beam having an energy in excess of $14.7 \mathrm{meV}$. This shift in composition will depend upon the incoming neutron flux as well as its energy, since higher-energy neutrons may induce several rotational transitions. The consequences of such a change are twofold. First, the total cross section that is $\Sigma_{Q} \propto \int d \omega S(Q, \omega)$ will now become dominated by inelastic intensities arising from 


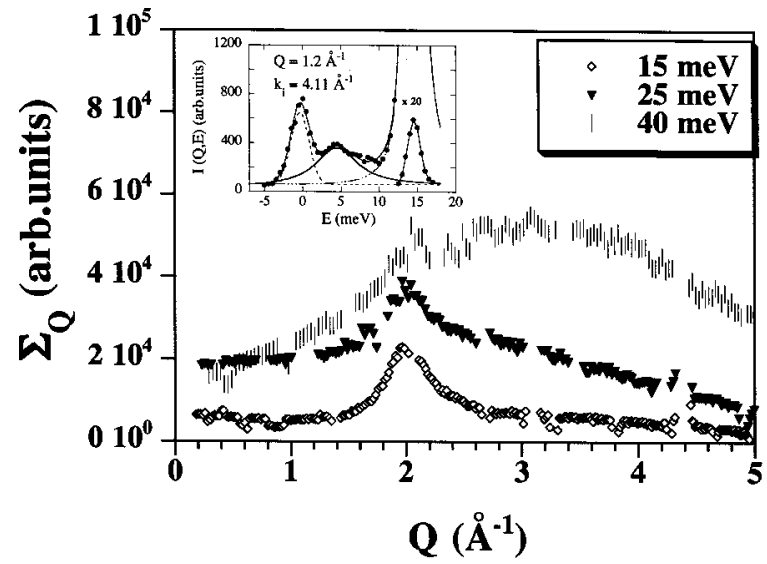

FIG. 1. Dependence of the observed cross section $\Sigma_{Q}$ $\propto \int_{i}^{e} d \omega S(Q, \omega)$ upon the upper integration limits that are given in the inset at the right-hand side. The inset at the left shows a spectrum for $Q=1.2 \AA^{-1}$, and serves to compare the intensity of the low-frequency region that comprises quasielastic scattering (dashes), a collective excitation peak (solid), and that corresponding to a $p \rightarrow o$ transition (dash-dots).

rotational excitations that are far stronger than the lowenergy part $E \leqslant 14.7 \mathrm{meV}$ of the spectrum (see the inset $^{6}$ of Fig. 1) that is related to $D_{m}(Q)$. In fact, the dynamic structure factor for individual molecule scattering is now given by

$$
f_{1}(Q)=\int d \omega S(Q, \omega)^{m o l},
$$

where the integrand can be calculated in closed form for the
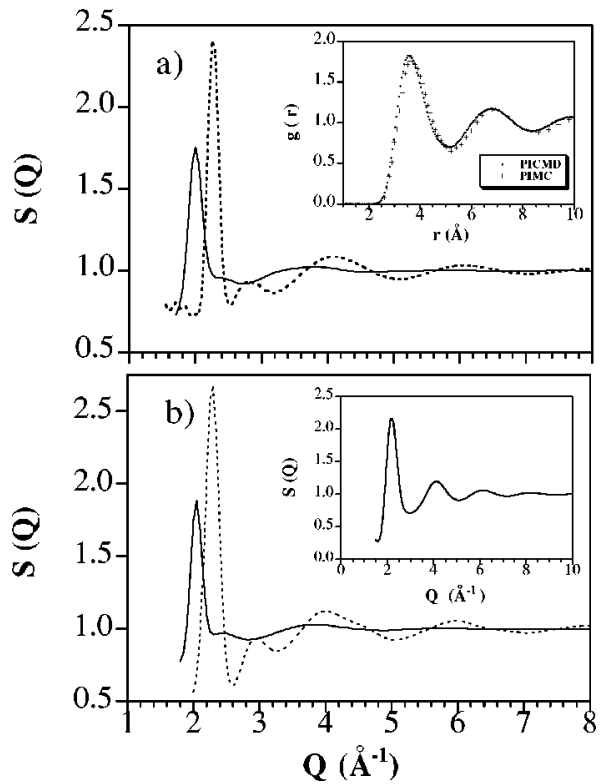

FIG. 2. A comparison between results for the $S(Q)$ static structure factor calculated by PIMC (Ref. 12) (upper, solid line) and that arising from the PICMD simulations (lower, solid line). The dotted lines depict results for the "classical" liquids. The inset in the upper frame shows a comparison between the $g(r)$ 's calculated from both PIMC and PICMD methods and that in the lower frame shows the static structure factor corresponding to a lower-density, stable liquid.

case where only rotational transitions are excited by the incoming neutron since the incident energy is lower than the first-excited vibrational state $\left(\hbar \omega_{v i b}=516 \mathrm{meV}\right)$ and the resulting expression reads ${ }^{7}$

$$
\begin{aligned}
S(Q, \omega)^{m o l} \propto & \left(\frac{M \beta}{\hbar^{2} Q^{2}}\right)^{1 / 2} \sum_{K=0,2,4, \ldots} p_{K}\left[\left(3 b^{(+)}+b^{(-)}\right)^{2} \sum_{K^{\prime}=0,2,4, \ldots}\left(2 K^{\prime}+1\right) \exp \left\{-\frac{M \beta}{\hbar^{2} Q^{2}}\left(E_{K^{\prime}}-E_{K}+\frac{\hbar^{2} Q^{2}}{4 M}-\hbar \omega\right)^{2}\right\}\right. \\
& \times \sum_{l=\left|K^{\prime}-K\right|}^{K^{\prime}+K}(2 l+1)\left(\begin{array}{ccc}
K^{\prime} & K & l \\
0 & 0 & 0
\end{array}\right)^{2} j_{l}^{2}\left(Q r_{d} / 2\right)+3\left(b^{(+)}-b^{(-)}\right)^{2} \sum_{K^{\prime}=1,3,5, \ldots}\left(2 K^{\prime}+1\right) \\
& \left.\times \exp \left\{-\frac{M \beta}{\hbar^{2} Q^{2}}\left(E_{K^{\prime}}-E_{K}+\frac{\hbar^{2} Q^{2}}{4 M}-\hbar \omega\right)^{2}\right\} \sum_{l=\left|K^{\prime}-K\right|}^{K^{\prime}+K}(2 l+1)\left(\begin{array}{rrr}
K^{\prime} & K & l \\
0 & 0 & 0
\end{array}\right)^{2} j_{l}^{2}\left(Q r_{d} / 2\right)\right],
\end{aligned}
$$

$$
p_{K}=\exp \left(-E_{K} / k_{B} T\right) / \sum_{K} \exp \left(-E_{K} / k_{B} T\right)
$$

where $\beta=\left(k_{B} T\right)^{-1}, M$ is the molecular mass, $p_{K}$ are thermal population factors, the spin-dependent scattering lengths are $b^{(+)}=1.04 \times 10^{-14} \mathrm{~m}, b^{(-)}=4.74 \times 10^{-14} \mathrm{~m}$, and $j_{l}()$ are spherical Bessel functions with arguments given in terms of the equilibrium internuclear distance $r_{d}=0.741 \AA$. Scattering from $\mathrm{p}-\mathrm{H}_{2}$ for neutron beams with energies exceeding $E_{K}=14.7 \mathrm{meV}$ will thus include intensities arising from para $\rightarrow$ ortho transitions that have associated $j_{l}^{2}\left(Q d_{e} / 2\right)$ with $l$ $>0$ form factors. The resulting molecular form factor $f_{1}(Q)$ will thus show a nontrivial $Q$ dependence that is also dependent upon the incident neutron energy. This means that the obtention of information concerning the liquid structure will be critically dependent upon the relative strength of the latter compared to the liquid structure function $D_{m}(Q)$. A vivid example of such effects is shown in Fig. 1 that depicts experimental data for $\Sigma_{Q}$ derived from an inelastic neutronscattering experiment ${ }^{6,8}$ where the experimentally accessible quantity was the double-differential cross section. An esti- 

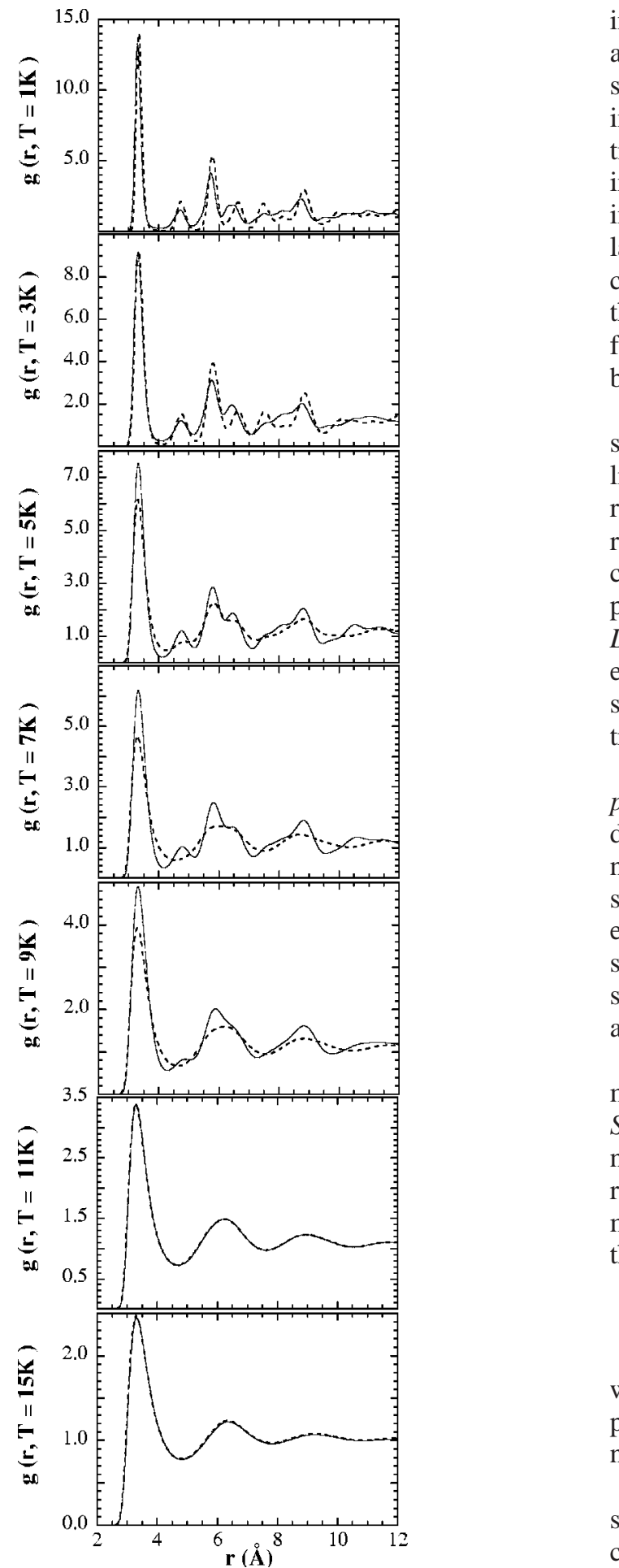

FIG. 3. A set of radial distribution functions corresponding to the melting of a stabilized fcc crystal (solid lines) and freezing of a liquid (dashes).

mate of the contributions of the rotational cross section is then made by integrating over energy transfers the measured double-differential cross section. The data shown in Fig. 1 provide a clear indication of the dependence of $\Sigma_{Q}$ on the integration range for a beam of $E_{i}=40 \mathrm{meV}$ neutrons, and show that the main peak in $D_{m}(Q)$ gets buried below the intensity arising from rotational excitations that also exhibits a rather marked dependence with wave vector. This poses severe difficulties to any attempt to derive direct structural information by standard diffraction means. In fact, a diffraction experiment that usually involves hot neutron beams having incident energies of a few tenths of an $\mathrm{eV}$ measures an integral over energy transfers that will contain intensities related to transitions to higher rotational levels. This can be clearly seen in data reported in Ref. 9 for $p-\mathrm{H}_{2}$ that evidence that the correction needed to account for the rotational form factors is far larger than the sought $D_{m}(Q)$ which can barely be seen as small shoulder in $\Sigma_{Q}$.

In contrast, the dependence of the single-differential cross section with the incident neutron energy is far less severe for liquid deuterium which from $E_{i}=2 \mathrm{meV}$ upwards shows a rather mild behavior. ${ }^{4}$ This comes as a consequence of the relatively weak intensities of the ortho $\rightarrow$ para transitions compared to that of the quasielastic and low-energy inelastic parts of the spectrum, which are the main contributors to $D_{m}(Q)$ as can be seen in spectra reported in Ref. 10. This enables the derivation of $D_{m}(Q)$ from the $\Sigma_{Q}$ measured by standard diffraction procedures since it is now the main contributor to the sought signal. ${ }^{11}$

An experimental estimate for the liquid structure factor of $p-\mathrm{H}_{2}$ (Ref. 8) came as a by-product of a study that dealt with dynamical properties. This means that the experiment was not optimized for the accurate determination of the liquid structure factor, which would require rather different sampleenvironment conditions. The results drawn in Fig. 1 of Ref. 8 show that the statistical accuracy of the measured liquid structure factor leaves much to be desired and calls for an accurate determination of this quantity.

A recent paper by Zoppi et al. ${ }^{12}$ reports on a path-integral monte carlo (PIMC) calculation of the static structure factor $S(Q)$, of liquid hydrogen. The quantity actually simulated is not directly amenable to experiment but should rather be regarded as a structure factor for the molecular centers of mass related to the experimentally accessible quantity through

$$
D_{m}(Q)=f_{2}(Q)[S(Q)-1],
$$

where $f_{2}(Q)$ is an intermolecular structure factor that depends upon the degree of orientational correlation between neighboring molecules.

Rather than considering actually calculated quantities such as the $g(r)$ radial distributions, the referred authors compare their PIMC results for $S(Q)$ with both the experimental estimate and the PICMD and classical liquid calculations reported in (Ref. 8). From this, the authors conclude that both the experiment and the calculation are flawed, while the authors own PIMC results are deemed to be reliable, on the basis of earlier results obtained for liquid deuterium ${ }^{11}$ and hydrogen. ${ }^{9}$

The quantity to compare concerning data from computer simulations is the $g(r)$ pair distribution function rather than $\mathrm{S}(\mathrm{Q})$ since these are quantities actually calculated while the latter may be subjected to significant truncation effects. The comparison drawn as an inset of Fig. 2(a) shows differences 
between $g(r)$ 's arising from PIMC and PICMD, which are small and explainable by the somewhat different thermodynamic state points used in both calculations as well as by the larger Trotter number used in our calculations (100 versus 32 of Zoppi et al. ${ }^{12}$ ). The differences in $S(Q)$ are thus attributable to the different inversion procedures used to deal with heavily truncated data. We made use of direct Fourier transform $^{8}$ while Zoppi et al. use an extrapolation of $g(r)$ to large distances by means of the formula suggested by Verlet. ${ }^{13}$ To enable a direct comparison of both sets of data we have followed the same procedure employed by Zoppi et al. ${ }^{12}$ The large- $r$ part of the $g(r)$ 's have been fitted to a decaying sine function ${ }^{12,13}$ and the fitted function extrapolated to $r=30 \AA$ previous to Fourier inversion. The results given in Fig. 2 show that the $S(Q)$ arising from PICMD has its main peak at $2.05 \AA^{-1}$ and a height of 1.88 and that from PIMC has its maximum at $2.00 \AA^{-1}$ and a height of 1.76 , a difference which is again compatible with that already observed for the $g(r)$ 's. At any rate, the physical soundness of such a difference is difficult to assess due to the rather approximate nature of the extrapolation procedure when applied to Lennard-Jones-like systems as it is explicitly stated in Ref. 13.

Concerning the calculations on "classical" $\mathrm{H}_{2}$ one should be aware that are dealing with liquids in the verge of crystallization that would map to the case of a Lennard-Jones system below the triple point. This makes the simulation results to be heavily dependent upon the thermodynamic conditions and thermal history of the sample. The simulation of the "classical" liquid reported in Ref. 8 corresponds to a reduced density ( $\rho=0.022 \AA^{-3}$ versus $0.024 \AA^{-3}$ for the PICMD), a value chosen in order to prevent crystallization. Under such conditions, the liquid modeled using the Goldman-Silvera potential ${ }^{5}$ shows a reversible freezing transition of about $13 \mathrm{~K}$ as can be inferred from data displayed in Fig. 3. The data just referred show that crystal nucleation and growth takes place within the simulation cell for temperatures of about $11 \mathrm{~K}$ and below. The final crystal structure attained upon subsequent cooling stages is significantly different from that corresponding to an ordered fcc structure taken as an initial reference of the heating runs, probably due to the coexistence of more than one crystal structure when freezing. ${ }^{14}$ At any rate, the relevant point here concerns the height of the classical $g(r)$ that can only reach values as high as that of 4 reported in Ref. 12 if the simulated liquid coexists with a sizable fraction of crystallites. The use of a lowerdensity liquid is therefore needed if one wants to compare the $S(Q)$ of both liquids rather than relating experimental and PICMD results to that of an inhomogeneous mixture. To compare with data shown in Ref. 12 we have carried out inversions of $g(r)$ data for the classical liquid as described by one of us ${ }^{3}$ and that shown in Ref. 12 using the same approach as before. The results given in Fig. 2 depict a structure factor with a peak at $2.30 \AA^{-1}$ having a height of 2.68 . Data taken from Ref. 12 yielded values of $2.25 \AA^{-1}$ and 2.40 , respectively. Again, the differences in position with respect to the reported value ${ }^{12}$ of $2.26 \AA^{-1}$ can be attributed mostly to details concerning the extrapolation procedure. The $S(Q)$ of data corresponding to the lower-density liquid is given as an inset in Fig. 2(b). Its position at $2.20 \AA^{-1}$ and its height of 2.16 come somewhat below those of the previous two cases and it is explainable as a density effect.

Zoppi's main criticism to our data concerns peak heights that, as shown here are critically dependent upon extrapolation and inversion procedures. In contrast, the difference in peak positions between classical and quantum liquids seems to be a far more reliable measure of the extent of quantum effects in these liquids. As a matter of fact, it suffices to compare data for liquid $\mathrm{D}_{2}$ (Ref. 11) with those for hydrogen to see that the main effect of the quantum degrees of freedom is to stabilize a liquid with a lower density than that which would correspond to a full classical liquid. More specifically, data for liquid $\mathrm{D}_{2}$ (Ref. 11) for a thermodynamic state close to that explored for $p-\mathrm{H}_{2}$ shows a $S(Q)$ with maxima at about $2.13 \AA^{-1}$ and a height of about 2.2. ${ }^{11}$ This corresponds to a liquid having a density about $15 \%$ higher than that of liquid $p-\mathrm{H}_{2}$, and therefore constitutes an intermediate case between the real liquid $p-\mathrm{H}_{2}$ and its classical analog.

In conclusion, PIMC and PICMD data yield rather close results when quantities resulting from simulations are considered and processed in the same way.

Work was supported in part by DGICYT (Spain), Grant No. PB98-0673-C02-01. K.K. acknowledges the ACT-JSF funds (Japan).

\footnotetext{
${ }^{1}$ M. Ross, Rep. Prog. Phys. 48, 1 (1985).

${ }^{2}$ F. Gygi and G. Galli, Phys. Rev. B 65, 220102 (2002); R. Redmer et al., ibid. 63, 233104 (2001); L.A. Collins et al., ibid. 63, 184110 (2001).

${ }^{3}$ K. Kinugawa, Chem. Phys. Lett. 292, 454 (1998).

${ }^{4}$ W. D. Seifert, Euratom Report No. EUR-4455-d, 1970 (unpublished); H. Würz, Kernforschung Karlsruhe Report No. KFK1697, 1973 (unpublished); Alternatively, see J.R. Granada, V.H. Gillete, and R.E. Mayer, Physica B 156-157, 164 (1989).

${ }^{5}$ I.F. Silvera, Rev. Mod. Phys. 52, 393 (1980).

${ }^{6}$ F.J. Bermejo et al., Phys. Rev. B 60, 15154 (1999).

${ }^{7}$ S. W. Lovesey, Theory of Neutron Scattering from Condensed Matter (Oxford Science, Oxford, U.K., 1986), p. 274.
}

${ }^{8}$ F.J. Bermejo et al., Phys. Rev. Lett. 84, 5359 (2000).

${ }^{9}$ M. Zoppi, M. Celli, and A.K. Soper, Phys. Rev. B 58, 11905 (1998).

${ }^{10}$ M. Mukherjee et al., Phys. Rev. B 57, R11 031 (1998); F.J. Bermejo et al., ibid. 47, 15097 (1993).

${ }^{11}$ M. Zoppi et al., Phys. Rev. Lett. 75, 1779 (1995); Also M. Zoppi et al., Phys. Rev. E 54, 2773 (1996).

${ }^{12}$ M. Zoppi, M. Neumann, and M. Celli, Phys. Rev. B 65, 092204 (2002).

${ }^{13}$ L. Verlet, Phys. Rev. 165, 201 (1968).

${ }^{14}$ P.R. ten Wolde, M.J. Ruiz-Montero, and D. Frenkel, Phys. Rev. Lett. 75, 2714 (1995). 